Technical and Operational Feasibility, for the business analysis, of running an Autopista

$$
\text { AIM }
$$

Evaluate the feasibility to run an Autopista Ferroviaria (AF)

with Iberina track gauge from a technical and operational point of view in order to

analyse an optimal business model

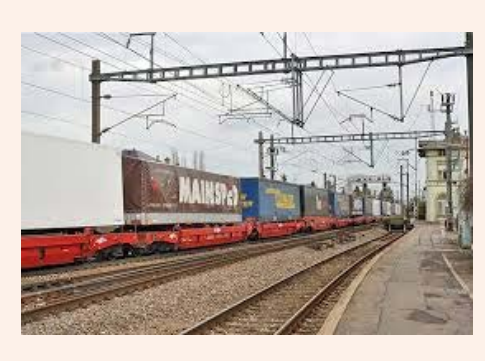
KEY WORD

AF: combined transport in which semitrailers are transported by train in a shuttle service

\section{TECHNICAL FEASIBILITY}

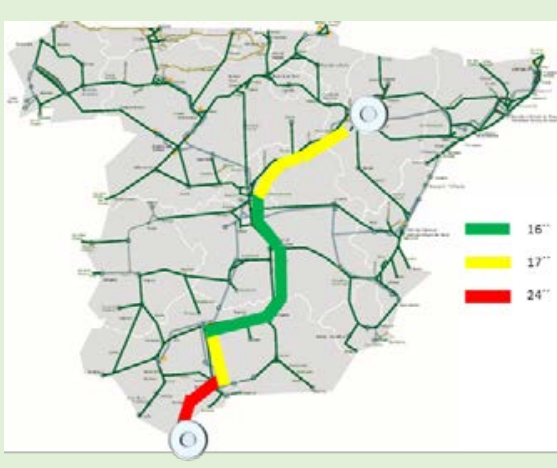

Maximum Slopes

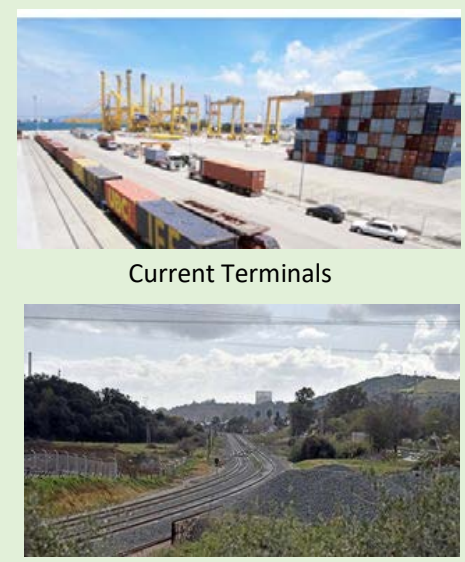

Minimum Siding Length (550 m.)
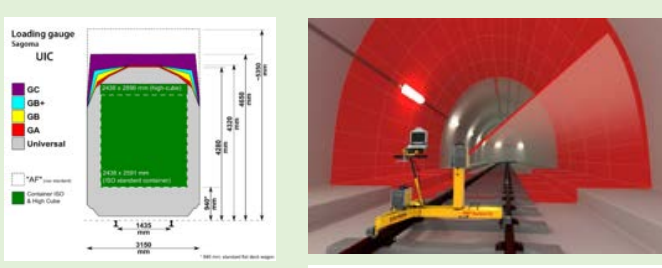

Clearance gauge

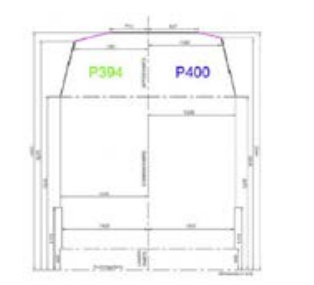

Technical Characteristics and its Implications

\begin{tabular}{|c|c|c|c|}
\hline Features & $\begin{array}{c}\text { Limitin } \\
\boldsymbol{g} \\
\text { factor }\end{array}$ & $\begin{array}{c}\text { Operation } \\
\text { al } \\
\text { Influence }\end{array}$ & $\begin{array}{c}\text { Economic } \\
\text { al } \\
\text { Influence }\end{array}$ \\
\hline Gauge & No & Yes & Yes \\
\hline Platforms & No & No & No \\
\hline Catenary & No & No & No \\
\hline $\begin{array}{c}\text { maximum Slope } \\
\text { minimum Axle- } \\
\text { load }\end{array}$ & No & Yes & Yes \\
\hline Siding length & No & Yes & Yes \\
\hline
\end{tabular}

\section{OPERATIONAL FEASIBILITY}

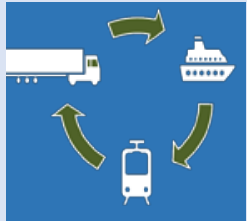

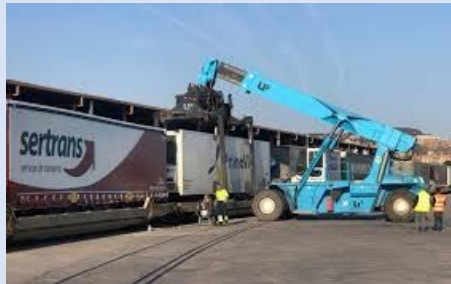

Reachstaker operating

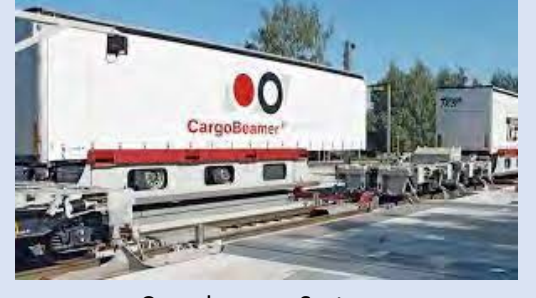

Cargobeamer System

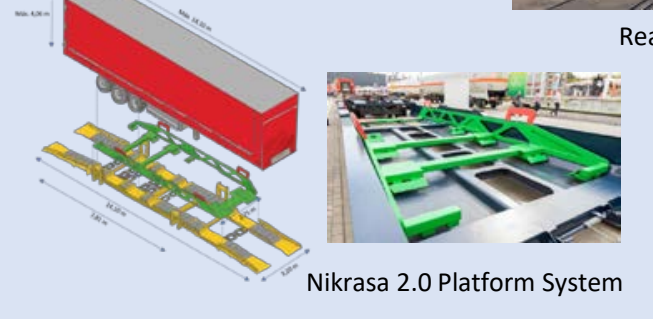

\begin{tabular}{c|c|c|c|}
$\begin{array}{c}\text { Locomotive } \\
\text { Gross Tons Towed }\end{array}$ & $16 "$ & $17^{\prime \prime}$ & $24^{\prime \prime}$ \\
\hline 253 & 1.180 & 1.130 & 860 \\
\hline
\end{tabular}

\begin{tabular}{|l|l|l|r|}
253 & 1.180 & 1.130 & 860 \\
\hline
\end{tabular}

\begin{tabular}{ll|l|l|l|}
253 DT* (special draw hook) & 2.130 & 2.040 & 1.550 \\
\hline
\end{tabular} \begin{tabular}{|l|l|l|l|}
\hline EURO 4000 (335) Diesel & 1.490 & 1.410 & 1.060 \\
\hline EURO 4000 (335) Diesel DT & 2.680 & 2.540 & 1.910
\end{tabular}

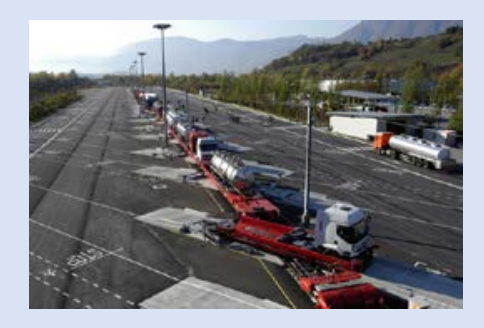

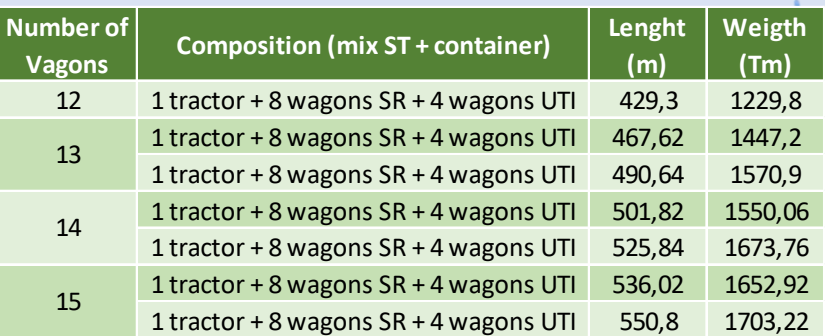

Length and train compositions

OperationalCharacteristics and its Implications

\begin{tabular}{|c|c|c|c|}
\hline Features & $\begin{array}{c}\text { Limiting } \\
\text { Factor }\end{array}$ & $\begin{array}{c}\text { Operational } \\
\text { Influence }\end{array}$ & $\begin{array}{c}\text { Economical } \\
\text { Influence }\end{array}$ \\
\hline Special Wagons & No & Yes & Yes \\
\hline Op $\quad$ Future Research
\end{tabular}

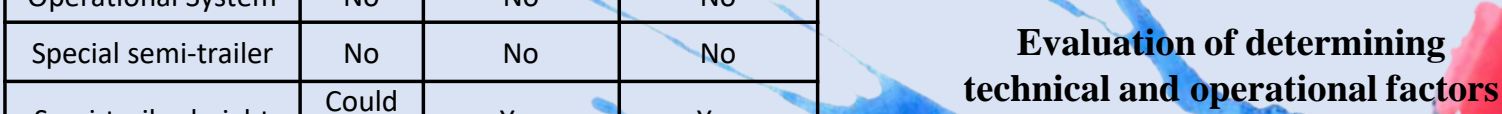
\begin{tabular}{|c|c|c|c|c|c|}
\hline Semi-trailer height & $\begin{array}{c}\text { Could } \\
\text { be }\end{array}$ & Yes & Yes & in order to validate and optimize
\end{tabular}

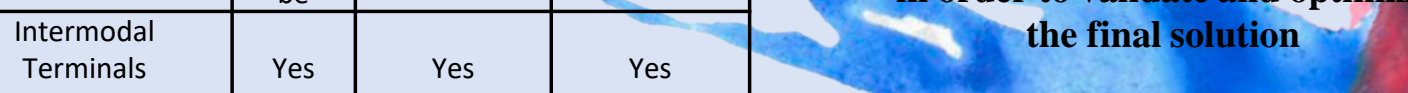

